\title{
Analysis of structures and epitopes of a novel secreted protein MYR1 in Toxoplasma gondii
}

\author{
Jian Zhou, Gang Lu and Shenyi He
}

Department of Parasitology, Shandong University School of Medicine, Jinan, China

\begin{abstract}
Toxoplasma gondii (Nicolle et Manceaux, 1908) is an obligate intracellular apicomplexan parasite and can infect warmblooded animals and humans all over the world. Development of effective vaccines is considered the only ideal way to control infection with $T$. gondii. However, only one live vaccine is commercially available for use in sheep and goats. Thus more effective antigenic proteins are searched for. In the present study we report a novel protein by secreted T. gondii termed Myc regulation 1 (MYR1). The physical and chemical characteristics, epitopes, hydrophilicity and functional sites of MYR1 were analysed by multiple bioinformatic approaches. The 3D models of MYR1 proteins were constructed and analysed. Furthermore, liner B-cell epitopes and T-cell epitopes of MYR1 protein and SAG1 were predicted. Compared to SAG1, MYR1 with good B-cell epitopes and T-cell epitopes had a potentiality to become a more successful vaccine against $T$. gondii. The bioinformatics analysis of MYR1 proteins could laid the foundation for further studies of its biological function experimentally and provide valuable information necessary for a better prevention and treatment of toxoplasmosis.
\end{abstract}

Keywords: Myc regulation, PTM sites, bioinformatic analysis, vaccine, toxoplasmosis

Toxoplasma gondii (Nicolle et Manceaux, 1908) is an opportunistic intracellular protozoan parasite that can infect a broad range of mammalian and avian hosts, includ humans (Chen et al. 2003, Nardoni et al. 2011, Zhou et al. 2011, Tian et al. 2012). According to epidemiological surveys, there was a wide distribution and high prevalence of T. gondii in many areas of the world (Tenter et al. 2000, Meng et al. 2012).

Acute infections in pregnant women can lead to hydrocephalus, miscarriage, blindness and mental retardation (Kravetz and Federman 2005). Infection with T. gondii may cause encephalitis because of reactivation of latent cysts in immunodeficient patients (Frenkel and Escajadillo 1987, Luft and Remington 1992), and T. gondii is major opportunistic pathogen in HIV-infected patients (Dziadek et al. 2011).

Toxoplasmosis causes abortions, stillbirths and neonatal deaths in livestock, leading to serious economic losses, and infected livestock is a major source of infection to humans (Bhopale 2003, Bai et al. 2012). The high incidence and severe or lethal damages of toxoplasmosis clearly show the need for the development of a more effective vaccine (Hoseinian et al. 2011).

Many studies showed that DNA vaccines could be an excellent solution because the vaccines could elicit long-lasting humoral and cell-mediated immunity, as well as provide protection against parasitic infections (Alarcon et al. 1999). The challenge for researchers is to identify and construct novel vaccines against $T$. gondii by selecting relevant proteins of this parasite as the ideal antigens. Then such antigens would be presented in an appropriate matrix to the host so they induce a strong and long-lasting immune response that provides protection against $T$. gondii (see Hiszczynska-Sawicka et al. 2011). So the study of DNA vaccine against the parasite is particularly important.

c-Myc is a key transcription factor that regulates critical host cell processes such as cell cycle progression, cell metabolism and apoptosis (Dang et al. 1997, 1999). By applying a flow cytometry-based selection to infected mouse cells expressing green fluorescent protein fused to c-Myc (c-Myc-GFP), Franco et al. (2016) isolated mutant tachyzoites defective in this host c-Myc up-regulation. Whole-genome sequencing of three such mutants led to the identification of Myc regulation 1 (MYR1; Gene ID: TGGT1_254470) as essential for c-Myc induction.

According to Franco et al. (2016), MYR1 is a novel protein that plays a critical role in how $T$. gondii delivers effector proteins to the infected host cell and that this is crucial to virulence. Also, MYR1 is necessary for full virulence (Franco et al. 2016). However, the protein's structures, function and basic characteristics are not clear. In the present study, bioinformatic approaches were used to analyse the structure, physical and chemical characters, structure and epitopes of the MYR1 protein. 

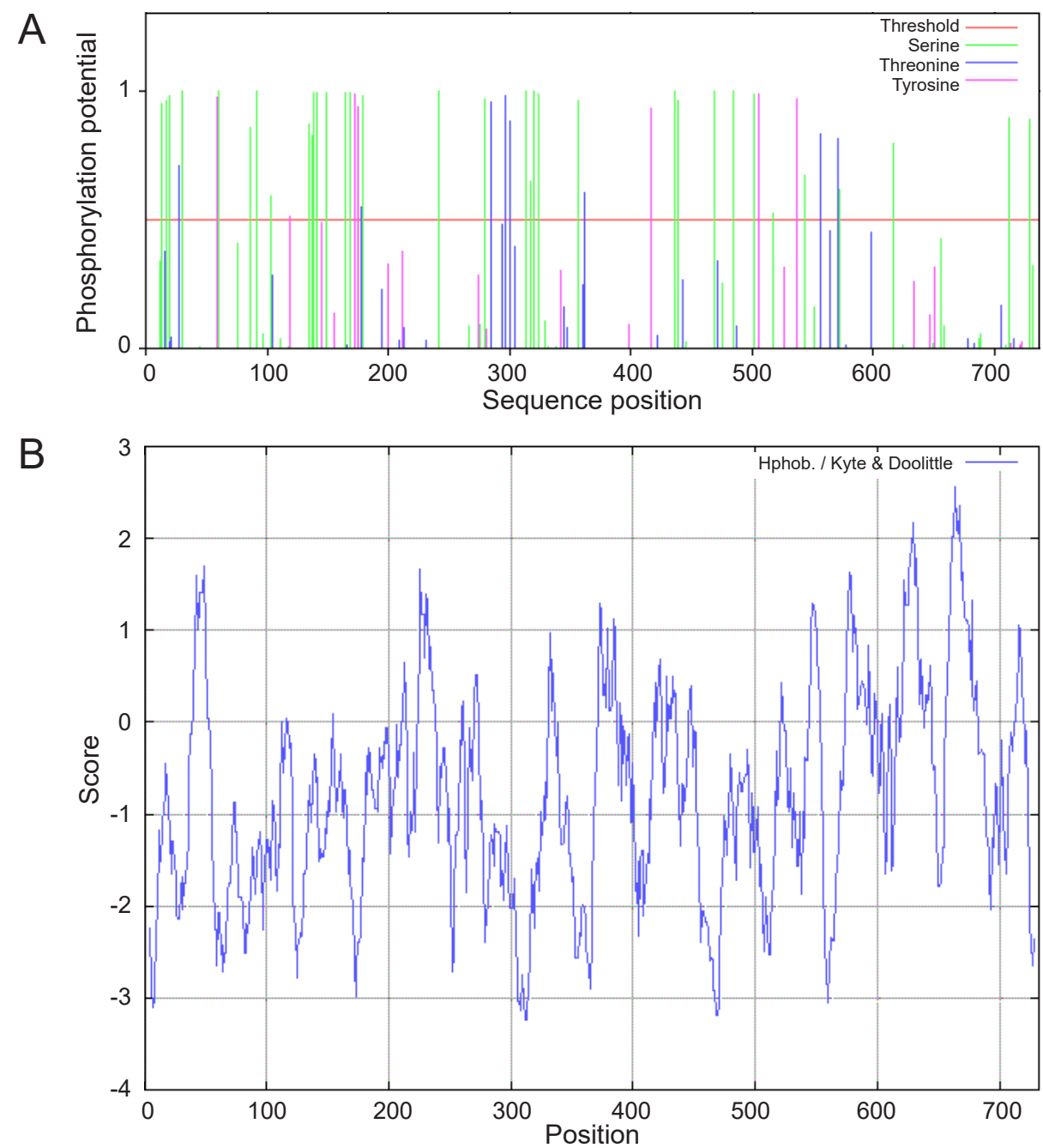

Fig. 1. NetPhos 2.0 Server was used to analyse the phosphorylation sites and ProtScale was used to predict the hydrophilicity/hydrophobicity of the MYR1 protein. A - analysis of the phosphorylation sites of MYR1; B - hydrophilicity/hydrophobicity of MYR1.

Previous report showed that MYR1 N-terminal and C-terminal domains localise to the parasitophorous vacuole membrane and western blotting showed that the expressed proteins can be recognised by anti-human c-Myc antibodies (Franco et al. 2016). In addition, the results indicated that antigenic index of MYR1 was better than SAG1. Moreover, MYR1 had more significant surface probability than SAG1 in the analysis result. All the positive results showed MYR1 protein can be a vaccine candidate against $T$. gondii. This report aimed at laying the foundation for the further study of the function and DNA vaccine of MYR1 protein.

The protein sequence of MYR1 of $T$. gondii was obtained from ToxoDB 10.0 (http://toxodb.org/toxo/). So far, apart from Franco et al. (2016), there have been no reported studies about the MYR1. In the present study, we used Prot-Param (http://web.expasy.org/protparam/) to predict protein molecular weight, isoelectric point, amino acid composition, instability index, aliphatic index and grand average of hydropathicity of MYR1. The amino acids sequence of MYR1 contains 732 residues, with a weight of $80.5 \mathrm{kD}$ and its theoretical $\mathrm{pI}$ is 5.13. The total number of negatively charged residues (Asp + Glu) is 117 whereas total number of positively charged residues (Arg + Lys) is 90. The instability index (II) is computed to be 42.56. This classifies the MYR1 protein as stable. The Aliphatic index is 61.19 and the Grand average of hydropathicity (GRAVY) is -0.817 .

Protein Post-Translational Modification (PTM) plays a significant role in cellular control mechanism (Lee et al. 2009). NetPhos 2.0 Server (http://www.cbs.dtu.dk/services/NetPhos/) was used to analyse the phosphorylation sites and CSS-Palm Online Service (http://csspalm.biocuckoo. org/online.php) was used to analysis the acylation sites. We found 49 phosphorylation sites (Ser 34, Thr 8, Tyr 7) (Fig. 1) and 5 acylation sites (position 201, 248, 336, 343 and 581) on the MYR1 sequence, suggesting that there will be 54 potential PTM sites on the sequence. MYR1 may regulate protein function through these sites, thereby affecting the protein activity.

Analysis of amino acid hydrophilicity/hydrophobicity could help understand the protein folding and interaction sites. It also could be used to predict the protein secondary structure and antigenic epitopes. Hydrophobicity is the main power of protein folding. Analysis of amino acid hydrophilicity/hydrophobicity is the first step in under- 
A
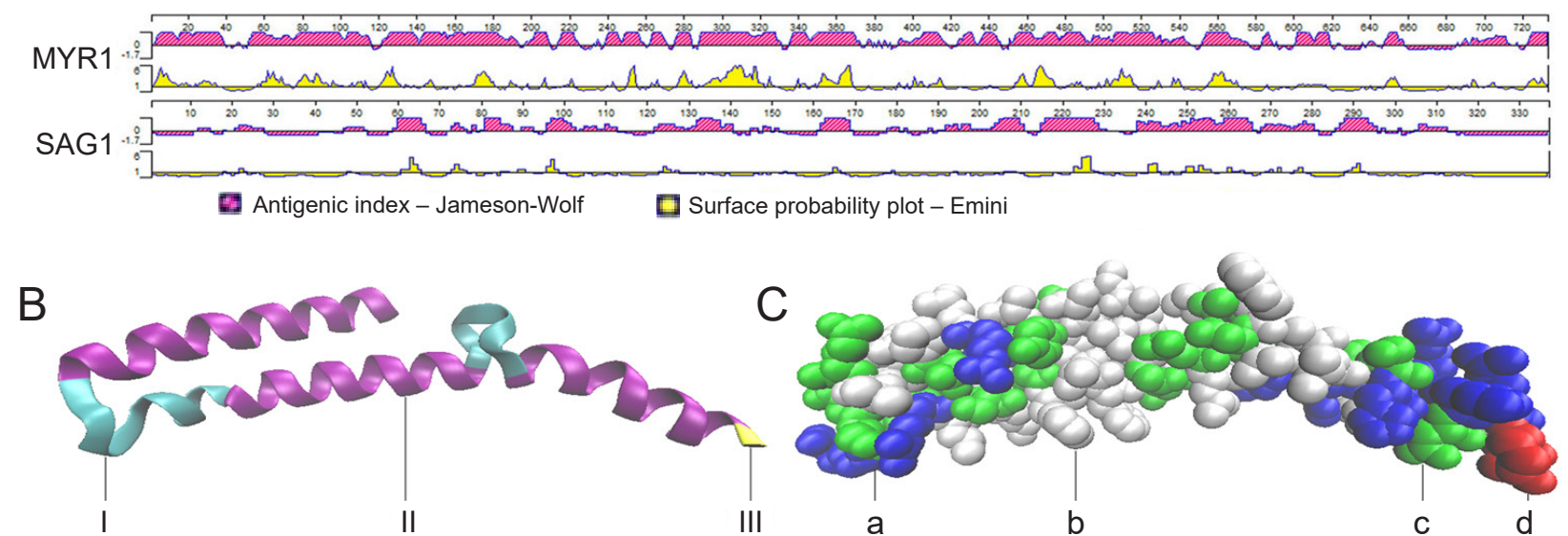

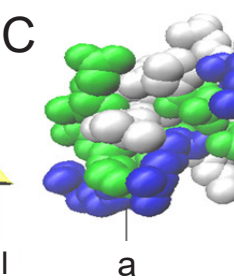

a

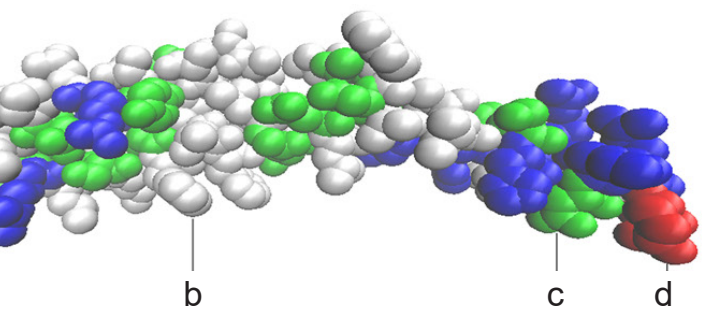

Fig. 2. A - linear-B cell epitopes of MYR1 and SAG1 predicted by the DNASTAR software in antigenic index and surface probability rules; $\mathbf{B}$ - prediction the secondary structures of MYR1 by DNASTAR software (I- turn; II - $\alpha$-helix; III - coil); C - 3D model constructed for MYR1 protein ( $\mathrm{a}$ - basic, $\mathrm{b}$ - nonpolar, $\mathrm{c}$ - polar, $\mathrm{d}$ - acidic).

standing protein folding, which could offer evidence for prediction of protein secondary structure and could be used for analysis of protein interaction sites - prediction of antigenic epitopes as an important step in the analysis of transmembrane proteins (Bai et al. 2012). We used ProtScale (http://web.expasy.org/cgi-bin/protscale/protscale.pl) to predict the hydrophilicity/hydrophobicity of the MYR1. The results show that the sequence has multiple hydrophilic regions and that there are also some classical high hydrophilic domains sucha as: 40-53aa, 224-238aa, 371-387aa, 544-553aa, 573-594aa, 620-644aa, 656-681aa and 713719aa (Fig. 1).

The linear B-cell epitopes of MYR1 were analysed and compared with SAG1 using DNASTAR software. The peptides that have good satisfactory flexibility and surface probability indicate positive antigenicity.

As shown in Fig. 2, SAG1, a good vaccine candidate protein, had excellent antigenic index and surface probability. The prediction result indicated that antigenic index of MYR1 was better than SAG1. Moreover, MYR1 had more significant surface probability than SAG1 in the analysis result. The online service IEDB was used to analyse T-cell epitopes of MYR1. The half maximal inhibitory concentration (IC50) values of peptides binding to the major histocompatibility complex (MHC) class II molecules of MYR1 were predicted with the online service. The T-cell epitopes on MYR1 that were identified by bioinformatic analyses were predicted to have the ability to bind strongly to MHC class II molecules.

Table 1 shows the minimum percentile ranks of each MHC II alleles on MYR1. A lower value of IC50 indicated higher affinity, which may mean a better T-cell epitope (Table 1). Obviously, compared to SAG1, MYR1 had much lower value of IC50 on the whole, which demonstrated that the latter could be an excellent T-cell antigen. In short, MYR1 with good B-cell epitopes and T-cell epitopes had great potentiality to become a successful vaccine against T. gondii.

The secondary structure of a protein makes a significant impact on the epitopes. The $\alpha$-helix and $\beta$-folded have a higher chemical bonds energy, which can be more solid
Table 1. IC50 values for MYR1 and SAG1 binding to MHC class II molecules obtained using the immune epitope database ${ }^{\mathrm{a}}$.

\begin{tabular}{lcccc}
\hline \multirow{2}{*}{ MHC II Allele $^{\mathrm{b}}$} & \multicolumn{2}{c}{ Start-Stop $^{\mathrm{c}}$} & \multicolumn{2}{c}{ Percentile Rank } \\
\cline { 2 - 5 } & SAG1 & MYR1 & SAG1 & MYR1 \\
\hline \multirow{2}{*}{ HLA-DRB1*01:01 } & $12-26$ & $663-677$ & 0.88 & 0.05 \\
& $35-49$ & $633-647$ & 2.74 & 0.96 \\
H2-IAb & $26-40$ & $207-221$ & 2.15 & 1.68 \\
& $297-313$ & $206-220$ & 2.81 & 1.74 \\
H2-IAd & $21-35$ & $621-635$ & 0.34 & 1.35 \\
& $168-182$ & $367-381$ & 1.22 & 0.88 \\
H2-IEd & $14-28$ & $179-193$ & 18.45 & 10.98 \\
& $34-48$ & $328-342$ & 30.62 & 11.47 \\
\hline
\end{tabular}

a the immune epitope database (http://tools.immuneepitope.org/mhcii); the prediction was run for three times; ${ }^{\mathrm{b}} \mathrm{H} 2$-IAb, H2-IAd and H2-IEd alleles are mouse MHC class II molecules; HLA-DRB1*01:01 allele is a human MHC class II molecule; ${ }^{\mathrm{c}}$ we chose 15 amino acids for analysis each time; ${ }^{\mathrm{d}}$ low percentile rank - high level binding, high percentile rank - low level binding, IC50 values - percentile rank.

to maintain the structure of a protein, more difficult to interact with antibodies, usually stay in the interior of the protein, and generally not act as epitopes. The random coil is looser, prone to distortion, hovering and exposed on the surface of the protein and may be a potential epitope. The secondary structures of MYR1 protein was predicted using DNASTAR_Lasergene.v7.1. (Fig. 2).

Three-dimensional structure of protein can be used to study the binding of antigens and antibodies. Biological function of proteins depends largely on the spatial structure of proteins; prediction of tertiary structures is the ultimate goal of predicting a protein's structure. It is of great significance to understand the structures of proteins and then to understand the relationship between structures and functions. In the present study, 3D model of MYR1 protein was constructed by SWISS-MODEL (Guex and Peitsch 1997, Schwede et al. 2003), a protein structure server on the website http://swissmodel.expasy.org/, which is considered to predict protein $3 \mathrm{D}$ structures that have many amino acids. VMD is a molecular visualisation software for displaying, animating and analysing large biomolecular systems using 3D graphics. VMD software was used to read stand- 
ard Protein Data Bank files and display the corresponding structure (Humphrey et al. 1996, Guex and Peitsch 1997, Schwede et al. 2003, Guex et al. 2009) (Fig. 2).

In conclusion, the present study showed the information of structure and basic characteristics of MYR1 protein. Taking account of the predicted results, we can draw a conclusion that MYR1 is a potential antigen protein of strong antigenicity, thus it may be a DNA vaccine candi- date against affection by $T$. gondii. The present study laid the foundation for further research on its biological function experimentally, and provided a theoretical basis for the screening of effective DNA vaccine candidate components against infection with $T$. gondii.

Acknowledgements. This work was supported, in part, by grants from the National Natural Science Foundation of China (Grant Nos. 81071373 and 81271857).

\section{REFERENCES}

Alarcon J.B., Waine G.W., McManus D.P. 1999: DNA vaccines: technology and application as anti-parasite and anti-microbial agents. Adv. Parasitol. 42: 343-410.

Bai Y., He S., Zhao G., Chen L., Shi N., Zhou H., Cong H., ZhaO Q., ZhU X.Q. 2012: Toxoplasma gondii: bioinformatics analysis, cloning and expression of a novel protein TgIMP1. Exp. Parasitol. 132: 458-464.

Bhopale G.M. 2003: Development of a vaccine for toxoplasmosis: current status. Microbes Infect. 5: 457-462.

Chen H., Chen G., Zheng H., Guo H. 2003: Induction of immune responses in mice by vaccination with liposome-entrapped DNA complexes encoding Toxoplasma gondii SAG1 and ROP1 genes. Chin. Med. J. (Engl.) 116: 1561-1566.

DANG C.V., LEwIS B.C. 1997: Role of oncogenic transcription factor c-Myc in cell cycle regulation, apoptosis and metabolism. J. Biomed. Sci. 4: 269-278.

Dang C.V., Resar L.M., Emison E., Kim S., Li Q., Prescott J. E., Wonsey D., Zeller K. 1999: Function of the c-Myc oncogenic transcription factor. Exp. Cell Res. 253: 63-77.

Dziadek B., Gatkowska J., Brzostek A., Dziadek J., Dzitko K., Grzybowski M., Dlugonska H. 2011: Evaluation of three recombinant multi-antigenic vaccines composed of surface and secretory antigens of Toxoplasma gondii in murine models of experimental toxoplasmosis. Vaccine 29: 821-830.

Franco M., Panas M.W., Marino N.D., Lee M.C., Buchholz K.R., Kelly F.D., Bednarski J.J., Sleckman B.P., PourMAND N., Boothroyd J.C. 2016: A novel secreted protein, MYR1, is central to Toxoplasma's manipulation of host cells. mBio 7: e02231-15.

Frenkel J.K., Escajadillo A. 1987: Cyst rupture as a pathogenic mechanism of toxoplasmic encephalitis. Am. J. Trop. Med. Hyg. 36: 517-522.

Guex N., Peitsch M.C. 1997: SWISS-MODEL and the Swiss-PdbViewer: an environment for comparative protein modeling. Electrophoresis 18: 2714-2723.

Guex N., Peitsch M.C., Schwede T. 2009: Automated comparative protein structure modeling with SWISS-MODEL and Swiss-PdbViewer: a historical perspective. Electrophoresis 30 Suppl. 1: S162-S173.
Hiszczynska-Sawicka E., Oledzka G., Holec-Gasior L., Li H., Xu J.B., Sedcole R., Kur J., Bickerstaffe R., StankIEWICZ M. 2011: Evaluation of immune responses in sheep induced by DNA immunization with genes encoding GRA1, GRA4, GRA6 and GRA7 antigens of Toxoplasma gondii. Vet. Parasitol. 177: 281-289.

Hoseinian K. K., Ghaffarifar F., D’Souza S., Sharifi Z., DALimi A. 2011: Evaluation of the immune response induced by DNA vaccine cocktail expressing complete SAG1 and ROP2 genes against toxoplasmosis. Vaccine 29: 778-783.

Humphrey W., Dalke A., Schulten K. 1996: VMD: visual molecular dynamics. J. Mol. Graph. 14: 33-38, 27-28.

Kravetz J.D., Federman D.G. 2005: Toxoplasmosis in pregnancy. Am. J. Med. 118: 212-216.

Lee T.Y., Hsu J.B., Chang W.C., Wang T.Y., Hsu P.C., Huang H.D. 2009: A comprehensive resource for integrating and displaying protein post-translational modifications. BMC Res. Notes 2: 111.

Luft B. J., Remington J.S. 1992: Toxoplasmic encephalitis in AIDS. Clin. Infect. Dis. 15: 211-222.

Meng M., He S., Zhao G., Bai Y., Zhou H., Cong H., Lu G., ZHAO Q., ZHU X. Q. 2012: Evaluation of protective immune responses induced by DNA vaccines encoding Toxoplasma gondii surface antigen 1 (SAG1) and 14-3-3 protein in BALB/c mice. Parasit. Vectors 5: 273.

Nardoni S., Angelici M.C., Mugnaini L., Mancianti F. 2011: Prevalence of Toxoplasma gondii infection in Myocastor coypus in a protected Italian wetland. Parasit. Vectors 4: 240.

Schwede T., Kopp J., Guex N., Peitsch M.C. 2003: SWISS-MODEL: an automated protein homology-modeling server. Nucl. Acids Res. 31: 3381-3385.

Tenter A.M., Heckeroth A.R., Weiss L.M. 2000: Toxoplasma gondii: from animals to humans. Int. J. Parasitol. 30: 1217-1258.

Tian Y.M., Dai F.Y., Huang S.Y., Deng Z.H., Duan G., Zhou D.H., Yang J.F., Weng Y.B., Zhu X.Q., Zou F.C. 2012: First report of Toxoplasma gondii seroprevalence in peafowls in Yunnan Province, Southwestern China. Parasit. Vectors 5: 205.

Zhou P., Chen Z., Li H.L., Zheng H., He S., Lin R.Q., Zhu X.Q. 2011: Toxoplasma gondii infection in humans in China. Parasit. Vectors 4: 165.

Cite this article as: Zhou J., Lu G., He S. 2016: Analysis of structures and epitopes of a novel secreted protein MYR1 in Toxoplasma gondii. Folia Parasitol. 63: 028. 